\title{
Advancing Pharmacy Service using Big Data - Are We Fully Utilising the Big Data's Potential Yet?
}

\author{
Hui-Qi See ${ }^{1}$, Jin-Ning Chan ${ }^{1}$, Shu-Jin Ling ${ }^{1}$, Shang-Cheng Gan ${ }^{1}$, Chee-Onn Leong ${ }^{1,2}$, Chun-Wai Mai ${ }^{1,2}$ \\ ${ }^{1}$ School of Pharmacy, International Medical University, Bukit Jalil, Kuala Lumpur, Malaysia. \\ ${ }^{2}$ Center for Cancer and Stem Cell Research, International Medical University, Bukit Jalil, Kuala Lumpur, Malaysia.
}

Received, March 29, 2018; Revised, June7, 2018; Accepted, June 20, 2018; Published, June 21, 2018.

\begin{abstract}
Big data is anticipated to have large implications in clinical pharmacy, in view of its potential in enhancing precision medicine and to avoid medication error. However, it is equally debatable since such a powerful tool may also disrupt the need of pharmacist in healthcare industry. In this article, we commented the contribution of Big Data in various aspects of clinical pharmacy including advancing pharmaceutical care service, optimising drug supplies, managing clinical trials, and strengthening pharmacovigilance. The future direction of the usage of Big Data related to clinical pharmacy will be discussed.
\end{abstract}

This article is open to POST-PUBLICATION REVIEW. Registered readers (see "For Readers") may comment by clicking on ABSTRACT on the issue's contents page.

\section{INTRODUCTION}

The term "Big Data" is defined as the massive data sets which are complicated and far beyond the ability of the conventional data analysis tools in capturing, assimilating, storing and interpreting the data within an appreciable time. Big Data can be further characterised by three key components namely, data volume (i.e vast amount of structured and unstructured data), data variety (i.e. data homogeneity) and data velocity (i.e. rate of data sets expansion). [1,2]

The rapid development in information and communication technologies in addition to the increasing affordability of electronic devices has significantly contributed to the growth of data from various sources. This has posed unique opportunities and great challenges in fully exploiting opportunities Big Data can offer. The well-known applications of Big Data today are the ability to use information to understand consumer demand and purchasing pattern for retail business.[3] With the expected growth of healthcare data volume to reach zettabytes $\left(10^{21}\right)$, there is an increase in the awareness and innovation of garnering data for drug discovery, precision medicine and patient-centred pharmacy practices with personalised approaches to prevention, early detection, treatment and optimum management.[2] The examples of Big Data application are the electronic medical record (EMR) and clinical decision support such as the First Databank and the use of Big Data Analysis in predicting epidemic tropical diseases. Although the growth in this area is still modest, it is undoubtedly a highly potential field to shift the paradigm of current pharmacy practices towards personalised care approaches and better pharmaceutical care [3-4].

In this article, we commented the contribution of Big Data in various aspects of clinical pharmacy. Among them include the implication on advancing pharmaceutical care service, accelerating clinical trials, and strengthening pharmacovigilance, specifically on how big data may be useful for pharmacists to provide better pharmaceutical care. We discussed the possible challenges and how can we move forward with other healthcare professionals in this digital era

\section{ADVANCING PHARMACEUTICAL CARE SERIVCE}

By collecting and integrating the health-related data from various trustworthy sources, Big Data can produce an extensive population health statistics on medication related indicators related to clinical outcomes and adverse drug events. The population statistics will enable identification of high-risk patient for early interventions. In addition, EMR of patients can also be used to predict patient outcomes by determining the best treatment decision [3] The utilization of advanced machine learning and artificial intelligence (AI),

Corresponding Author: Dr. Chun-Wai Mai PhD, BPharm (Hons), International Medical University, No.126, Jalan Jalil Perkasa 19, Bukit Jalil, 57000 Kuala Lumpur, Malaysia; Email:chunwai_mai@imu.edu.my 
such as the IBM Watson [5], has demonstrated significant benefits in the healthcare delivery system. Predictive modelling using real-time data was shown to reduce adverse drug reactions, improve disasters management in hospitals and reduce re-admissions for patients with specific disease.[6] The goal of personalised care plan can be achieved through the use of the decision support tool as it tailors the use of pharmacological agent according to the patientspecific parameters such as serum biomarkers and metabolic profile. Besides, real time patient's clinical response will also allow healthcare provider to notice the changes in critically-ill patient status and employ appropriate action plan. Ultimately, Big Data can assist the clinical benchmarking database to improve clinical pharmacy service.[3]

Big Data has proven to improve patient's medication compliance. Express Scripts is an integrated medication management tool through pharmacy claims processing, medication delivery, and personal medication inventory. With the integration of data analytics, it can be used to predict the compliancy of patients to the prescribed medicines, possibility to be addicted to opioids or those who are defrauding the health system based on consumer demographics and attributes, disease states, prescribed drugs, and refill rates. Express Scripts reported that with this support system, the rate of non-adherence to curative treatment was reduced from $8.3 \%$ to $4.8 \%$, saving the patients approximately US $\$ 30,000$ in drug costs. An integrated delivery system in the northern California was also developed to improve medication adherence using online patient portal.[7]

In the outpatient setting, Big Data plays even bigger role in measuring the quality of care and patient's outcomes. A recent study conducted in the Greater Manchester, United Kingdom, demonstrated that Big Data can be used to benchmark the performance of general practitioners and identify the sources of waste within a workflow. By comparing the pattern of prescription and hospital admissions, the study has concluded that Big Data has optimized resources utilization within the region. A similar effort was also being pioneered in the Pharmacy Quality Solutions in the US to use Big Data to track the performance of the pharmacy and from there to choose the preferred pharmacy networks. [7-9]

Besides, predictive analysis and the 'Internet of Things' (IoTs) may provide detailed demand forecasting at the level of point-of-sale and ensure the in-store availability of drugs in healthcare providers. Moreover, predictive analysis also helps in optimising finished inventory, which provide inventory budget optimisation and safety stock level recommendations. This can help in reducing the inventory cost and to avoid wastage of expired medication. Big Data also allows the insights of scoring models for vendor quality, cost and stability and hence support pharmacist to secure long-term high-quality supplier partners. $[10,11]$

\section{ACCELERATING CLINICAL TRIALS}

The emergence of Big Data has accelerated the drug development and drug repurposing in pharmaceutical industries.[12] The process of drug development requires a clear pathophysiology of the disease. The generation of huge genomics data resulted from various "big science" initiatives, such as the Human Genome Project and the Cancer Genome Atlas project, has undoubtedly accelerated our understanding of various human diseases. This, in turn, has led to the rapid translation of the knowledge into discovery of biomarkers and the development of novel targeted therapies.[13,14]

The analysis, collection and processing of large volume of structured and unstructured biomedical data and surveys collected by researchers and pharmaceutical industries will build a better predictive modelling that could help to accelerate the drug discovery process. This success is a result of multidimensional Big Data analysis, artificial intelligence and machine learning in determining novel drug structure to target a selected pathological condition. $[13,14]$

In addition to accelerating drug discovery, Big Data can also accelerate drug repurposing. Drug repurposing is the re-positioning the available clinical drugs for new indications.[2,6] This approach can dramatically reduce the cost, time and risk of introducing a new drug to the patients. One of the approaches for drug repositioning is the "Disease Focus" method which utilises the varieties of 'omics' data for repurposing. For instance, by using the knowledge of phenotypic modulation by various drugs in the market, researchers are able to repurpose 58 potential drugs with a new indication for diabetes through analyses of the data obtained from genome wide association studies (GWAS), proteomic and metabolomics studies in diabetes patients. Out of the 58 drug candidates, nine of them, including niflumic acid, phenoxybenzamine and perhexiline, were shown to be highly effective against diabetes. By cross 
checking the data available on disease associated proteins, the data generated from various drug development projects and the data on the pathogenesis of the disease, it will help to streamline preclinical or clinical trials to establish the effectiveness of the repurposed drugs for the treatment of human diseases.[6]

In the era of Big Data, it is postulated that there is a potential switch in paradigm of the clinical trial sector from the prospective randomised controlled trial (RCT) to Big-data Clinical Trial (BCT). The current dominating research method, RCT solely compares the effectiveness of different interventions at the baseline without the detailed explanation of the association between the types of intervention and clinical outcomes comprehensively by considering all possible aetiologies and risk factors.[15] However, a biologically effective drug may not be the clinically effective drug. This problem arises due to the RCT experimental designed with strict protocol, inclusion and exclusion criteria may not resemble the reality conditions. In real life, patients are often complicated by comorbidities or unexpected events such as missing dose and delay in drug administration.[14] The discrepancies between the RCT and real world conditions have warranted the need of BCT, which is a comprehensive analysis of the overall samples instead of a smallsample subjects from a large population like RCT. This means that the clinical decision made based on BCT will be more clinically relevant. However, there are major challenges to the implementation of BCT including patients' privacy, policy and reliability of information. Nevertheless, BCT promise to reshape the future of clinical research should these issues be addressed adequately.[15,16]

\section{STRENGTHENING}

\section{PHARMACOVIGILANCE}

Adverse Drug Reactions (ADRs) monitoring which is defined as adverse patient outcomes resulting from a medication, has become a challenging research problem that gradually gains attentions from the medical centres. The usage of Big Data has become a useful resource for adverse drug reactions monitoring primarily due to its sheer volume.[17] The appeal of Big Data is evident due to the ability to identify and evaluate associations between drugs, events, and risk factors is the basis of pharmacovigilance.[18]

Recently, abundant text accessible in healthrelated forums and social media along with the rich expression of public opinion have gained attraction from public health community to use social media for pharmacovigilance. Based on the insight that patients post regarding ADRs which mainly expressing negative sentiments, researchers start to investigate the effect of sentiment analysis features in locating ADR mentions. Evaluation results show that sentiment analysis features slightly improve ADR identification in tweet posts. Sentiment analysis features achieved a statistically significant $F$ measure increase from $72.14 \%$ to $73.22 \%$ in the Twitter part of an existing corpus using its reference and test samples. This study shows that addition of sentiment analysis features can slightly improve the performance of even a new ADR identification method. This improvement can be used as pharmacovigilance practice for clinical pharmacists because of the rapidly increasing popularity of using health forums and social media.[19]

\section{CHALLENGES OF BIG DATA}

Although big data is set to revolutionize the delivery of healthcare, several challenges exist. First, human barriers such as lack of big data and analytical skills are considered as the most challenging steps when utilising big data in healthcare. Organizations and pharmacies encounter shortage of qualified data scientists to utilize big data. Primary skill such as the knowledge in big data technologies and platforms and secondary skills including advanced in mathematics and statistics, predictive analytics and decision-making models are currently lacking in the training of healthcare professional. This may hinder the progress of implementing big data in pharmacy practice.[2]

Second, there are huge numbers of isolated databases exist today. Majority of them are difficult to determine the sources, reliability, availability and fitness of the data. In addition, highly valuable data can exist in semi-structured and unstructured form, e.g. health records and scientific literature. For structured data, there are often discrepancy in terms the data format, i.e. same type of data could be provided in different format or similar data field using the same vocabulary can have different meaning. For instance, different ontologies are developed to identify disease in different context. Duplicated records are another issues that need to be addressed.[2]

Thirdly is the patient confidentiality. As there is huge number of data collections stored online, cyber threats could happen and causes financial loss as well as decreased consumer confidence. 
Health organization must monitor patient information carefully by hiring qualified health informatics professionals to ensure security of patient databases.[20]

It is important to ensure the data do not fall into the wrong authority. Keeping the data safely and protect it away from wrong authority will be another difficult step faced by big data. Although the benefits brought by big data is tremendous, the coexist problems resulting from misuse would bring another opposite major issue.[8]

\section{FUTURE DIRECTION FOR PHARMACISTS}

It is expected that healthcare will slowly move towards digital modelling and thus Big Data has pivotal role in clinical pharmacy service. We summarise the potential support available for pharmacists and the potential benefit patients could receive from the Big Data intervention in healthcare (Figure 1).

It's exciting for clinical pharmacists to apply digital technology such as smart pillboxes that can improve patient's compliance in real time. There are also the in-store beacons that give signal or alarm to alert a pharmacist when an at-risk customer enters the pharmacy. McKinsey Global Institute estimates that Big Data strategies can generate up to $\$ 100$ billion profit for healthcare system. The ability of pharmaceutical companies to identify new potential drug can be improved by utilizing Big Data and develop them into effective medicines rapidly. Predictive modelling of biological processes and drugs that act safely and effectively on biological targets. It can be done easily by leveraging the diversity of available molecular and clinical data. More effective realtime monitoring for clinical trial could be in place and thus improves the clinical pharmacists' efficiency. Besides, adverse events and unnecessary delays can be avoided and trials are monitored in real time to identify safety or alarming operational signals. Instead of rigid data silos which are difficult to exploit, data are captured electronically and flow easily between discovery and clinical development as well as to external partners such as physicians and contract research organizations.[21].

\section{CONCLUSION}

Big Data is generated by rapid assimilation of technology from resources that range from simple to complex in their content. Health system pharmacy leaders must understand Big Data and its uses in order to help improving pharmacy practice model.[3] Clinical pharmacist is positioned to play important role partnering with other healthcare providers to increase access and improve the quality of patient care at the same time contributing towards controlling healthcare spend.[10] To date, clinical pharmacy is just the beginning to touch upon the iceberg of information available as compared to other pharmacy sectors. A multitude of other areas warrant further exploration on how Big Data can be used by pharmacists as the possibilities are endless. Challenges of Big Data that faced by pharmacy or pharmaceutical industry have to be identified and overcome completely for a brighter and better future. As health care information expands in its content and becomes more integrated, Big Data will be a valuable tool in enhancing the development of patient-centred clinical pharmacy services.

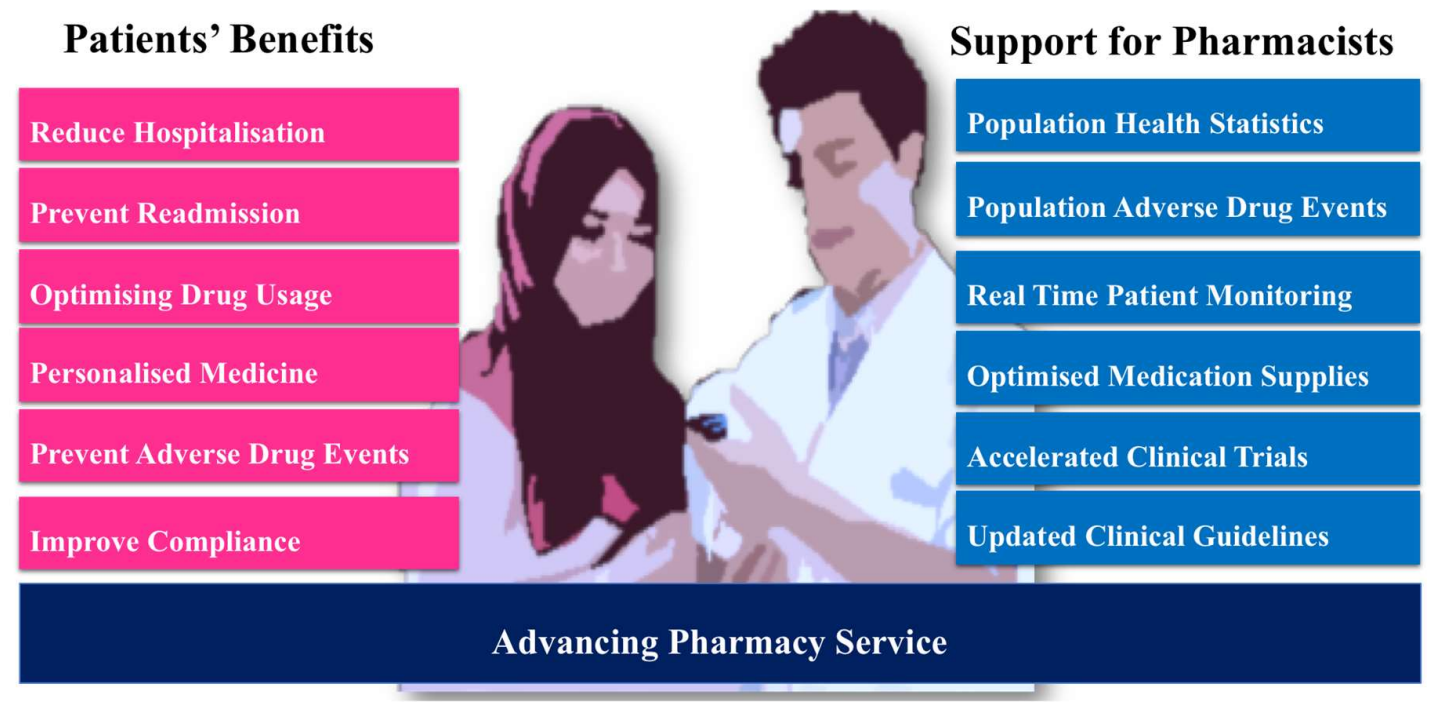

Figure 1. The Big Data supports available for pharmacists to advance the pharmacy service to patients. 


\section{ACKNOWLEDGEMENTS}

The authors wish to acknowledge the researchers in the Institute of Research, Development and Innovation, International Medical University for the critical comments on the manuscripts. There is no funding received for this manuscript.

\section{CONFLICTS OF INTEREST}

The authors declare no financial conflict of interest.

\section{REFERENCES}

1. Alharthi A, Krotov V, Bowman M. Addressing barriers to big data. Bus Horiz. 2017;60(3):285-92. doi:10.1016/j.bushor.2017.01.002

2. Taglang G, Jackson DB. Use of big data in drug discovery and clinical trials. Gynecol Oncol. 2016;141(1):17-23. doi:10.1016/j.ygyno.2016.02.022

3. Stokes LB, Rogers JW, Hertig JB, Weber RJ. Big Data: Implications for health system pharmacy. Hosp Pharm. 2016; 51(7):599-603. doi:10.1310/hpj5107-599.

4. Gianfredi V, Bragazzi NL, Nucci D, Martini M, Rosselli R, Minelli L, et al. Harnessing Big Data for Communicable Tropical and Sub-Tropical Disorders: Implications From a Systematic Review of the Literature. Front Public Health. 2018;6:90. doi:10.3389/fpubh.2018.00090.

5. Hoyt RE, Snider D, Thompson C, Mantravadi S. IBM Watson Analytics: Automating Visualization, Descriptive, and Predictive Statistics. JMIR Public Health Surveill. 2016 Oct 11;2(2):e157. doi:10.2196/publichealth.5810

6. Zhang M, Luo H, Xi Z, and Rogaeva E. Drug repositioning for diabetes based on 'omics' data mining. 2015; 10(5): e0126082. doi:10.1371/journal.pone.0126082.

7. Lawrence J. Could big data be the future of pharmacy? The Pharmaceutical Journal. 2017. Accessed from http://www.pharmaceuticaljournal.com/news-and-analysis/features/could-bigdata-be-the-future-of-pharmacy/20202637.article. Accessed 4 March 2018

8. Jones S. Leveraging of Power of Big Data in Community Pharmacies. SmartData Collective 2016. Available from https://www.smartdatacollective.com/leveragingpower-big-data-community-pharmacies/. Accessed 4 March 2018

9. Ma K, Smith HW, Chu C, Juarez DT. Big Data in pharmacy practice: Current use, challenges, and the future. Integr Pharm Res Pract. 2015;4(91-99). doi: 10.2147/IPRP.S55862.

10. Marques R, Gregório J, Mira Da Silva M, Lapão $\mathrm{LV}$. The promise of internet of things in healthcare: How hard is it to keep? Stud Health Technol
Inform. 2016; 228: 665-9. doi:10.3233/978-161499-678-1-665

11. Schreier G. The internet of things for personalized health. Stud Health Technol Inform. 2014; 200 : 22-31. doi:10.3233/978-1-61499-393-3-22

12. Wooller SK, Benstead-Hume G, Chen X, Ali Y, Pearl FMG. Bioinformatics in translational drug discovery. Biosci Rep. 2017;37(4). pii: BSR20160180. doi:10.1042/BSR20160180

13. Cha Y, Erez T, Reynolds IJ, Kumar D, Ross J, Koytiger G, Kusko R, Zeskind B, Risso S, Kagan E, Papapetropoulos S, Grossman I, Laifenfeld D. Drug repurposing from the perspective of pharmaceutical companies. Br J Pharmacol. 2018; 175(2):168-180. doi:10.1111/bph.13798.

14. Zhang R, Simon G, Yu F. Advancing Alzheimer's research: A review of big data promises. Int J Med Inform. $\quad 2017 \quad$;106: 48-56. doi:10.1016/j.ijmedinf.2017.07.002.

15. Wang SD. Opportunities and challenges of clinical research in the big-data era: from RCT to BCT. J Thorac Dis. 2013; 5(6):721-723. doi:10.3978/j.issn.2072-1439.2013.06.24

16. Zhang $Z$. Big data and clinical research: perspective from a clinician. $J$ Thorac Dis. 2014;6(12):1659-64. doi:10.3978/j.issn.20721439.2014.12.12

17. Sarker A, Ginn R, Nikfarjam A, O'Connor K, Smith K, Jayaraman S, Upadhaya T, Gonzalez G. Utilizing social media data for pharmacovigilance: A review. J Biomed Inform. 2015; 54:202-12. doi:10.1016/j.jbi.2015.02.004

18. Price J. What can Big Data offer the pharmacovigilance of orphan drugs? Clin Ther. 2016; 38: 2533-45. doi:10.1016/j.clinthera.2016.11.009.

19. Korkontzelos I, Nikfarjam A, Shardlow M, Sarker A, Ananiadou S, Gonzalez G. Analysis of the effect of sentiment analysis on extracting adverse drug reactions from tweets and forum posts. J Biomed Inform. 2016; 62:148-58. doi: 10.1016/j.jbi.2016.06.007

20. How Big Data is Affecting Pharmacy Practice. Available from: http://healthinformatics.uic.edu/resources/articles/ how-big-data-is-affecting-pharmacy-practice/ Assessed 8 June 2018.

21. Panahiazar M, Taslimitehrani V, Jadhav A, Pathak J. Empowering personalized medicine with Big Data and semantic web technology: Promises, challenges, and use cases. Proc IEEE Int Conf Big Data. 2014: 790-795. doi: 10.1109/BigData.2014.7004307. 